\title{
The impact of short daily hemodialysis on anemia and the quality of life in Chinese patients
}

\author{
J.L. Jiang ${ }^{1,2}$, W. Ren ${ }^{2}$, J. Song ${ }^{1}$, Q.L. Sun ${ }^{1}$, X.Y. Xiao ${ }^{1}$, X.Z. Diao ${ }^{2}$, Y.H. Huang ${ }^{2}$, L. Lan ${ }^{2}$ \\ P. Wang ${ }^{2}$ and Z. $\mathrm{Hu}^{1}$ \\ ${ }^{1}$ Department of Nephrology, Qilu Hospital, Shandong University, Jinan, China \\ ${ }^{2}$ Department of Nephrology, Anhui Provincial Hospital Affiliated to Anhui Medical University, Hefei, China
}

\begin{abstract}
Anemia is a frequent complication in hemodialysis patients. Compared to conventional hemodialysis (CHD), short daily hemodialysis (sDHD) has been reported to be effective in many countries except China. The aim of the present study was to determine whether SDHD could improve anemia and quality of life (QOL) for Chinese outpatients with end-stage renal disease. Twenty-seven patients ( 16 males/11 females) were converted from CHD to sDHD. All laboratory values were measured before conversion (baseline), at 3 months after conversion (sDHD1), and at 6 months after conversion (sDHD2). The patient's QOL was evaluated at baseline and 6 months after conversion using the Medical Outcomes Study 36-Item Short Form Health Survey (SF-36). Hemoglobin concentration increased significantly from $107.4 \pm 7.9 \mathrm{~g} / \mathrm{L}$ at baseline to $114.4 \pm 6.8 \mathrm{~g} / \mathrm{L}(\mathrm{P}<0.05)$ at SDHD1, and $118.3 \pm 8.4 \mathrm{~g} / \mathrm{L}(\mathrm{P}<0.001)$ at sDHD2 (Student paired $t$-test). However, the dose requirement for erythropoietin decreased from $6847.8 \pm 1057.3 \mathrm{U} /$ week at baseline to $5869.6 \pm 1094.6 \mathrm{U} /$ week $(\mathrm{P}<0.05)$ at sDHD2. Weekly stdKt/V increased significantly from $2.05 \pm 0.13$ at baseline to $2.73 \pm 0.20(P<0.001)$ at sDHD1, and $2.84 \pm 0.26(P<0.001)$ at sDHD2. C-reactive protein decreased from baseline to SDHD1 and SDHD2, but without statistically significant differences. Physical and mental health survey scores increased in the 6 months following conversion to sDHD. sDHD may increase hemoglobin levels, decrease exogenous erythropoietin dose requirements, and improve QOL in Chinese hemodialysis patients compared to CHD. A possible mechanism for improvement of clinical outcomes may be optimized management of uremia associated with the higher efficiency of sDHD.
\end{abstract}

Key words: Short daily hemodialysis; Anemia; Erythropoietin; Quality of life; End-stage renal disease

\section{Introduction}

Anemia and impaired quality of life (QOL) are frequent complications of end-stage renal disease (ESRD) in patients undergoing hemodialysis therapy and are caused by many factors. Hemodialysis patients often have anemia caused by inadequate synthesis of erythropoietin (EPO), and require therapy with exogenous recombinant human erythropoietin (rHuEPO) to maintain recommended hemoglobin levels. Many treatments to improve the anemia and QOL of patients with ESRD have been investigated $(1,2)$. Short daily hemodialysis (sDHD) was first described in 1968 by DePalma et al. (3) and has been widely used (4). In many countries, except for China, SDHD has been reported to be effective in hemodialysis patients (2,5-8), but there are few reports on its efficacy in managing anemia and QOL in Chinese patients undergoing hemodialysis. Because the practice patterns of hemodialysis and the body weight of patients in China differ from those in other countries, it is uncertain if SDHD could be as effective for Chinese patients undergoing hemodialysis as in other groups. The objective of the present study was to determine whether SDHD could indeed improve anemia and QOL in Chinese patients undergoing hemodialysis compared to conventional hemodialysis (CHD).

\section{Patients and Methods}

The subjects were stable outpatients with ESRD undergoing hemodialysis in the Anhui Provincial Hospital Hemodialysis Unit. They had been undergoing CHD using a native arteriovenous fistula or central venous cuffed dual-lumen catheter for 8 months or longer and were able

Correspondence: Z. Hu, Department of Nephrology, Qilu Hospital, Shandong University, 107\# Wenhuaxi Road, Jinan 250012, China. E-mail: huzhaophd2012@sina.com or jielong1013@sina.cn 
to visit the hospital for SDHD sessions. Twenty-seven patients (16 males/11 females), $46.8 \pm 13.4$ years of age (mean $\pm S D$; range: $26-70$ years) were enrolled. They were treated with $\mathrm{CHD}$ for $54.7 \pm 32.9$ months (range: 8-133 months) for $4 \mathrm{~h}, 3$ times per week, and then converted to SDHD for 2 h, 6 times per week. The causes of ESRD were glomerulonephritis $(n=13)$, diabetes mellitus $(n=6)$, hypertension $(n=4)$, polycystic kidney disease $(n=2)$, interstitial nephritis $(n=1)$, and connective tissue disease $(n=1$; Table 1$)$.

All patients gave written informed consent. There were no specific patient selection criteria. All patients underwent sDHD for 6 months (sDHD period) following a baseline observation period for 1 month ( $\mathrm{CHD}$ period). All hematological parameters were measured prior to the conversion from CHD to SDHD (baseline), at 3 months after the conversion (sDHD1), and at 6 months after the conversion (sDHD2).

rHuEPO was administered by subcutaneous injection maximally 3 times per week to maintain hemoglobin levels within the target range of $110-120 \mathrm{~g} / \mathrm{L}(11-12 \mathrm{~g} / \mathrm{dL})$. Iron was administered either orally (150-450 mg/day) or intravenously (50-150 mg/week) to maintain transferrin saturations greater than $20 \%$ or serum ferritin concentrations higher than $200 \mathrm{ng} / \mathrm{mL}$. Information on dosage and administration of other drugs (e.g., folate, vitamin B12) used concomitantly throughout the study was recorded. Serum albumin concentration and serum C-reactive protein (CRP) were measured throughout the study.

Blood flow rate ranged between 350 and $450 \mathrm{~mL} / \mathrm{min}$. Dialysate flow rate was $500 \mathrm{~mL} / \mathrm{min}$. High-flux polysulfone dialyzers and Fresenius 4008S (Fresenius Medical Care, Germany) dialysis machines were used for all patients. Hemodialysis conditions, including dialysis prescription and total weekly hemodialysis time, were the same for the $\mathrm{CHD}$ and sDHD periods. Weekly standard Kt/V (stdKt/V) was calculated to evaluate dialysis adequacy.

Patient QOL was evaluated at baseline and 6 months after conversion from $\mathrm{CHD}$ to SDHD using the Medical

Table 1. Baseline characteristics of patients.

\begin{tabular}{lc}
\hline Baseline characteristics & \\
\hline Patients (male/female) & $27(16 / 11)$ \\
Age (years, mean \pm SD) & $46.8 \pm 13.4$ \\
Duration of CHD (months, mean \pm SD) & $54.7 \pm 32.9$ \\
Etiology of ESRD & \\
Glomerulonephritis & 13 \\
Diabetes mellitus & 6 \\
Hypertension & 4 \\
Polycystic kidney disease & 2 \\
Other & 2 \\
\hline
\end{tabular}

CHD: conventional hemodialysis; ESRD: end-stage renal disease.
Outcomes Study 36-Item Short Form Health Survey (SF-36), and scores of general health perceptions, physical functioning, physical role functioning (role limitations due to physical health problems), bodily pain, vitality, mental health, social role functioning, and emotional role functioning (role limitations due to emotional health problems) were recorded.

Data are reported as means $\pm S D$. Statistical analysis was performed using the Student paired $t$-test for data measurements and the Wilcoxon test for ordered categorical data. $\mathrm{P}<0.05$ was considered to be significant.

\section{Results}

All patients tolerated SDHD well; no severe hemodialysis complications occurred in the course of this study. Four patients discontinued sDHD therapy before the study completion: 2 patients had difficulty visiting the hospital 6 days per week, 1 patient received a renal transplant, and 1 patient terminated SDHD because of the cost. The available data from these patients were used in all analyses.

Changes in all laboratory values after conversion from $\mathrm{CHD}$ to SDHD are summarized in Table 2. Hemoglobin concentration increased significantly from CHD to SDHD1 $(\mathrm{P}<0.05)$ and sDHD2 $(\mathrm{P}<0.001)$. However, the EPO dose requirement decreased from $\mathrm{CHD}$ to SDHD1 and sDHD2 $(P<0.05)$. No statistically significant differences in hemoglobin concentration or EPO dose requirement at sDHD1 and sDHD2 were observed. The serum albumin concentration increased significantly from $\mathrm{CDH}$ to sDHD1 and sDHD2 $(\mathrm{P}<0.001)$ but there was no statistically significant difference in serum albumin concentration at sDHD1 and sDHD2.

Serum ferritin increased from CHD to sDHD1 and sDHD2, however, without statistically significant differences. Transferrin saturations were consistently greater than $20 \%$. There were no statistically significant differences in transferrin saturation, folate, or vitamin B12 at any of the three periods. Doses of iron supplements were similar at all times and stable throughout the course of the study. The range of mean oral iron doses was 150 to $450 \mathrm{mg} /$ day, and the range of intravenous iron doses was 50 to $150 \mathrm{mg} /$ week. As a result, all subjects had adequate iron stores throughout the duration of the study. Weekly stdKt/V increased significantly from $\mathrm{CDH}$ to sDHD1 and sDHD2 $(\mathrm{P}<0.001)$. CRP decreased from $\mathrm{CHD}$ to sDHD1 and SDHD2, however, there were no statistically significant differences in CRP at any of the three periods.

As shown in Table 3, physical and mental health scores were improved after conversion to SDHD for 6 months. Scores for general health, physical functioning, bodily pain, vitality, and mental health increased $(P<0.05)$. However, there were no statistically significant differences in the scores for physical role functioning, social role functioning, and emotional role functioning. 
Table 2. Changes in laboratory values after conversion from $\mathrm{CHD}$ to SDHD.

\begin{tabular}{lccc}
\hline & CHD (baseline) & sDHD1 (3 months) & sDHD2 $(6$ months) \\
\hline $\mathrm{Hb}(\mathrm{g} / \mathrm{L})$ & $107.4 \pm 7.9$ & $114.4 \pm 6.8^{*}$ & $118.3 \pm 8.4^{* *}$ \\
EPO dose $(\mathrm{U} /$ week) & $6847.8 \pm 1057.3$ & $6358.7 \pm 1354.3$ & $5869.6 \pm 1094.6^{*}$ \\
Serum albumin $(\mathrm{g} / \mathrm{L})$ & $30.4 \pm 3.5$ & $39.2 \pm 4.7^{* *}$ & $41.2 \pm 4.4^{* *}$ \\
Serum ferritin $(\mathrm{ng} / \mathrm{mL})$ & $241.0 \pm 35.6$ & $244.6 \pm 37.9$ & $253.1 \pm 32.8$ \\
Transferrin saturation $(\%)$ & $26.4 \pm 4.1$ & $28.0 \pm 3.7$ & $27.6 \pm 3.9$ \\
Folate $(\mathrm{ng} / \mathrm{mL})$ & $13.59 \pm 3.31$ & $12.94 \pm 3.53$ & $13.95 \pm 3.44$ \\
Vitamin B12 $(\mathrm{pg} / \mathrm{mL})$ & $490.74 \pm 161.64$ & $463.91 \pm 169.30$ & $510.96 \pm 192.45$ \\
CRP $(\mathrm{mg} / \mathrm{L})$ & $6.2 \pm 3.3$ & $5.6 \pm 2.1$ & $5.8 \pm 2.7$ \\
Weekly stdKt/V & $2.05 \pm 0.13$ & $2.73 \pm 0.20^{* *}$ & $2.84 \pm 0.26^{* *}$ \\
\hline
\end{tabular}

Data are reported as means \pm SD. CHD: conventional hemodialysis; sDHD: short daily hemodialysis; Hb: hemoglobin concentration; EPO: erythropoietin; CRP: serum C-reactive protein; stdKt/V: standard index for dialysis efficiency. ${ }^{*} \mathrm{P}<0.05$ and ${ }^{* *} \mathrm{P}<0.001$ compared to $\mathrm{CHD}$ (Student paired $t$-test).

\section{Discussion}

Currently, the major hemodialysis technique in China is CHD. Many patients undergoing CHD have complications including anemia and impaired QOL. In the last several years, many studies have shown that SDHD could improve clinical outcomes in patients with ESRD compared to CHD. According to these studies, sDHD could improve the state of anemia, reduce the required dose level of EPO, and increase the QOL scores. However, those results were obtained mainly from hemodialysis patients in countries other than China.

This study shows that, compared to CHD, sDHD was associated with significantly higher hemoglobin concentration and lower EPO dose requirements, together with higher scores for physical and mental health-related QOL measures, in Chinese patients. Because of economic and other reasons, few patients in China are willing to accept sDHD, and at the beginning of this study, 27 patients were enrolled, but 4 patients terminated sDHD. In randomized

Table 3. Changes in the quality of life after conversion from $\mathrm{CHD}$ to sDHD.

\begin{tabular}{lcc}
\hline & $\begin{array}{c}\text { CHD } \\
\text { (baseline) }\end{array}$ & $\begin{array}{c}\text { sDHD } \\
(6 \text { months })\end{array}$ \\
\hline General health & $26.22 \pm 11.84$ & $37.43 \pm 15.51^{*}$ \\
Physical functioning & $50.65 \pm 13.51$ & $61.96 \pm 12.86^{*}$ \\
Physical role functioning & $31.52 \pm 22.88$ & $35.87 \pm 21.09$ \\
Bodily pain & $52.57 \pm 16.89$ & $63.96 \pm 19.81^{*}$ \\
Vitality & $31.09 \pm 12.79$ & $38.26 \pm 14.27^{*}$ \\
Mental health & $52.52 \pm 16.81$ & $63.48 \pm 18.50^{*}$ \\
Social role functioning & $30.07 \pm 11.81$ & $36.96 \pm 17.47$ \\
Emotional role functioning & $50.73 \pm 19.80$ & $59.44 \pm 28.35$ \\
\hline
\end{tabular}

CHD: conventional hemodialysis; sDHD: short daily hemodialysis. ${ }^{*} \mathrm{P}<0.05$ compared to CHD (Student paired $t$-test). controlled trials comparing SDHD with $\mathrm{CHD}$, patients who expressed greater willingness to participate were younger, less likely to smoke, more likely to have been hospitalized during the previous 12 months, less likely to have coronary artery disease, and less likely to be on a waiting list for a kidney transplant (9). In our study, it should be noted that each patient served as his or her own control to control for heterogeneity in the etiology of renal disease, which gave acceptable baseline values for assessing the effects of SDHD.

In many other studies, hemoglobin levels in patients treated with SDHD were higher than those in patients treated with CHD (5-8). However, some reports showed no significant change in hemoglobin levels in patients undergoing SDHD $(10,11)$. Several studies reported that dose requirements for EPO had decreased with SDHD therapy $(5-8,12-14)$. Koshikawa et al. (12) found that, in 14 patients with SDHD therapy, EPO was eliminated in 2 patients and its dose reduced in 5 patients. In the study by Ting et al. (14), requirements for EPO were reduced in $45 \%$ of patients after 12 months of follow-up. Our study confirms that SDHD increases hemoglobin levels and decreases EPO requirements. These findings are independent of differences in iron replenishment. According to a study by Goldfarb-Rumyantzev et al. (15), although requirements for EPO dosage trended down during SDHD and increased when $\mathrm{CHD}$ was resumed, there was no statistically significant difference between the SDHD and CHD periods. In this study, the serum albumin concentration increased significantly after changing from $\mathrm{CHD}$ to sDHD therapy. This may mean that hemoconcentration is a possible confounder for the increase in observed hemoglobin concentration or that SDHD may improve the nutritional status of dialysis patients. Further studies designed to verify this conjecture are needed.

The reasons for SDHD improving anemia in ESRD patients are not entirely clear. Anemia in patients with ESRD results from inadequate synthesis of EPO, iron deficiency, 
inflammation, and secondary hyperparathyroidism. Uremia-related EPO resistance is often associated with inflammation. Inflammation plays a key role in the hyporesponsiveness of erythropoiesis-stimulating agents (ESA) in hemodialysis patients who have sufficient iron and EPO. Possible mediators include cytokines such as interleukin-6 (IL-6), IL-10, insulin-like growth factor 1, and tumor necrosis factor- $\alpha(\mathrm{TNF}-\alpha)(16-18)$. In vitro, proinflammatory cytokines, including IL-1, IL-6, IL-10, interferon- $\gamma$, and TNF- $\alpha$, inhibit the growth of erythroid precursor cells and downregulate the expression of EPO receptor mRNA (16). Won et al. (17) found that IL-6 was a strong predictor of ESA hyporesponsiveness and an independent risk factor for resistance to ESA in hemodialysis patients without iron deficiency. Hamlett and Haragsim (19) reported that more frequent dialysis may have a beneficial effect on the inflammatory process that occurs in ESRD. More frequent (i.e., daily nocturnal) dialysis decreased plasma IL-6 levels and exogenous EPO requirements, and direct associations were found between EPO requirements, CRP $(R=0.62, P=0.001)$ and $I L-6(R=0.57, P=0.002)$ levels according to a study by Yuen et al. (20). They concluded that a possible mechanism for reducing EPO requirements was better control of inflammation, as manifested by decreased plasma IL-6 levels. In this study, CRP decreased from the CHD therapy period to the sDHD therapy period. However, the difference was not significant. Further studies are needed to measure proinflammatory cytokines in dialysis.

In the study by Suri et al. (21), sDHD resulted in increased dose delivery compared to CHD. There was a $20 \%$ increase in weekly stdKt/V with the sDHD regimen of 6 days/week for $2 \mathrm{~h}$ sessions, and sDHD was found to be the most efficient method of dose delivery. SDHD may reduce uremic toxin levels that inhibit erythropoiesis or simply provide a more normal physiological milieu for erythropoiesis by avoiding the peaks and troughs seen with CHD. In this study, weekly stdKt/V increased significantly from $2.05 \pm 0.13$ on conventional hemodialysis to $2.73 \pm 0.20(\mathrm{P}<0.001)$ at $\mathrm{sDHD1}$, and $2.84 \pm 0.26$ $(\mathrm{P}<0.001)$ at sDHD2. In our opinion, a possible mechanism for improvement of anemia and requirement for lower doses of EPO depends on optimized management of uremia with the higher efficiency of sDHD.

In the majority of trials, it was observed that sDHD improved physical and mental health-related QOL in patients with ESRD (2,22-25). Vos et al. (23) found that sDHD significantly improved "general health perception", a single item of the Kidney Disease QOL (KDQoL) questionnaire, as well as the KDQoL dimension scores "physical health" and "patient satisfaction". Recently, in a study by Finkelstein et al. (25), the SF-36 health survey was used to measure long-term effects of SDHD on healthrelated $\mathrm{QOL}$. They found that both the physical and mental component summary scores improved over a 12-month period in the total cohort analysis, indicating that SDHD was related to long-term improvements in both physical and mental health-related QOL measures. QOL improves with sDHD due to less severe dietetic rules, elimination of postdialysis asthenia, and fewer drug prescriptions. Short hemodialysis sessions ( $2 \mathrm{~h}$ daily) can be more easily integrated into professional, social, and family life. Our study demonstrates that SDHD is associated with significant improvement in QOL. SDHD therapy caused a large burden for the patients, as they had to visit the dialysis facility almost every day for SDHD. Despite the burden, there was no deterioration in QOL in this study.

Anemia causes tissue and organ hypoxia with a number of symptoms, signs, and physiological abnormalities. Among the common findings are daytime fatigue, disturbed sleep, and altered cognitive function. Anemia is universally present in this setting. The Kidney Disease Outcomes Quality Initiative clinical practice guidelines and clinical practice recommendations demonstrated that anemia in chronic kidney disease has some relevance to QOL (26). Several reports showed that sDHD improved anemia and QOL simultaneously $(6,7,12)$. Benz et al. (27) reported that the improvement in anemia reduced periodic limb movements in sleep, arousals from sleep, and sleep fragmentation while allowing for more restorative sleep and improved daytime alertness. Their findings may explain one mechanism for the improved QOL parameters reported in ESRD patients. In this study, hemoglobin concentration increased from 107.4 to $118.3 \mathrm{~g} / \mathrm{L}$. Meanwhile, the scores for general health, physical functioning, bodily pain, vitality, and mental health improved significantly. However, the observed improvement in QOL could have occurred because of more frequent visits to the hospital, optimized management of uremia, or other causes not addressed in this study and may not be related to anemia improvement.

A limitation of this study is that the SDHD therapy period was only 6 months and the parameters of secondary hyperparathyroidism were not recorded. Since the followup period was so short, we cannot be certain that the observed improvements persisted mid- and long-term. Because parameters of secondary hyperparathyroidism were not recorded, a mechanism for improvement of anemia by SDHD was not further identified. Further studies need to be designed to answer these questions.

In the present study, sDHD increased hemoglobin levels, decreased exogenous EPO dose requirements, and improved physical and mental health scores compared to $\mathrm{CHD}$ in Chinese hemodialysis patients. A possible mechanism for this improvement in clinical outcomes is optimized management of uremia with the higher efficiency of sDHD. Further studies are required to examine the relationship between anemia and QOL, and to explore the effect of other factors such as secondary hyperparathyroidism on anemia on long-term outcomes in patients with ESRD. Meanwhile, an important aspect for further study is whether patients would be willing to be dialyzed almost every day. 


\section{References}

1. Panichi V, Scatena A, Paoletti S, Migliori M. Impact of dialysis technique on renal anemia. Contrib Nephrol 2011; 171: 261-265, doi: 10.1159/000327341.

2. Punal Rioboo J, Sanchez-Iriso E, Ruano-Ravina A, Varela Lema ML, Sanchez-Guisande D, Gonzalez-Rodriguez L, et al. Short daily versus conventional hemodialysis quality of life: a cross-sectional multicentric study in Spain. Blood Purif 2009; 28: 159-164, doi: 10.1159/000227784.

3. DePalma JR, Pecker EA, Gordon A, Maxwell MH. A new compact automatic home hemodialysis system. Trans Am Soc Artif Intern Organs 1968; 14: 152-159.

4. Allen N, Schwartz D, Komenda P, Pauly RP, Zimmerman D, Tanna G, et al. International practice patterns and factors associated with non-conventional hemodialysis utilization. BMC Nephrol 2011; 12: 66, doi: 10.1186/1471-2369-12-66.

5. Lindsay RM, Heidenheim AP, Leitch R, Ryan H, Kroeker A, Peters $\mathrm{K}$, et al. Short daily versus long nocturnal hemodialysis. Daily/Nocturnal Dialysis Study Group. ASAIO J 2001; 47: 449-455, doi: 10.1097/00002480-200109000-00007.

6. Galland R, Traeger J, Arkouche W, Delawari E, Fouque D. Short daily hemodialysis and nutritional status. Am J Kidney Dis 2001; 37: S95-S98, doi: 10.1053/ajkd.2001.20758.

7. Punal J, Lema LV, Sanhez-Guisande D, Ruano-Ravina A. Clinical effectiveness and quality of life of conventional haemodialysis versus short daily haemodialysis: a systematic review. Nephrol Dial Transplant 2008; 23: 2634-2646, doi: $10.1093 / \mathrm{ndt} / \mathrm{gfn} 010$.

8. Klarenbach S, Heidenheim AP, Leitch R, Lindsay RM. Reduced requirement for erythropoietin with quotidian hemodialysis therapy. ASAIO J 2002; 48: 57-61, doi: 10.1097/00002480-200201000-00012.

9. Israni AK, Halpern SD, McFadden C, Israni RK, Wasserstein A, Kobrin S, et al. Willingness of dialysis patients to participate in a randomized controlled trial of daily dialysis. Kidney Int 2004; 65: 990-998, doi: 10.1111/ j.1523-1755.2004.00460.x.

10. Fagugli RM, Reboldi G, Quintaliani G, Pasini P, Ciao G, Cicconi B, et al. Short daily hemodialysis: blood pressure control and left ventricular mass reduction in hypertensive hemodialysis patients. Am J Kidney Dis 2001; 38: 371-376, doi: 10.1053/ajkd.2001.26103.

11. Fagugli RM, De Smet $R$, Buoncristiani $U$, Lameire $N$, Vanholder R. Behavior of non-protein-bound and proteinbound uremic solutes during daily hemodialysis. Am J Kidney Dis 2002; 40: 339-347, doi: 10.1053/ajkd.2002.34518.

12. Koshikawa S, Akizawa T, Saito A, Kurokawa K. Clinical effect of short daily in-center hemodialysis. Nephron Clin Pract 2003; 95: c23-c30, doi: 10.1159/000073015.

13. Traeger J, Galland R, Delawari E, Arkouche W, Hadden R. Six years' experience with short daily hemodialysis: do the early improvements persist in the mid and long term? Hemodial Int 2004; 8: 151-158, doi: 10.1111/j.1492-7535.2004.01089.x.

14. Ting GO, Kjellstrand C, Freitas T, Carrie BJ, Zarghamee S. Long-term study of high-comorbidity ESRD patients converted from conventional to short daily hemodialysis. Am J Kidney Dis 2003; 42: 1020-1035, doi: 10.1016/j.ajkd.2003.07.020
15. Goldfarb-Rumyantzev AS, Leypoldt JK, Nelson N, Kutner NG, Cheung AK. A crossover study of short daily haemodialysis. Nephrol Dial Transplant 2006; 21: 166175, doi: 10.1093/ndt/gfi116.

16. van der Putten K, Braam B, Jie KE, Gaillard CA. Mechanisms of disease: erythropoietin resistance in patients with both heart and kidney failure. Nat Clin Pract Nephrol 2008; 4: 47-57, doi: 10.1038/ncpneph0655.

17. Won HS, Kim HG, Yun YS, Jeon EK, Ko YH, Kim YS, et al. IL-6 is an independent risk factor for resistance to erythropoiesis-stimulating agents in hemodialysis patients without iron deficiency. Hemodial Int 2012; 16: 31-37.

18. Kim DH, Kim TY, Kim SM, Yoo SJ, Oh DJ, Yu SH. IGF-1 is an independent risk factor for anemia in diabetic pre-dialysis patients. Korean J Intern Med 2007; 22: 186-191, doi: 10.3904/kjim.2007.22.3.186.

19. Hamlett L, Haragsim L. Quotidian hemodialysis and inflammation associated with chronic kidney disease. Adv Chronic Kidney Dis 2007; 14: e35-e42, doi: 10.1053/ j.ackd.2007.03.006.

20. Yuen D, Richardson RM, Fenton SS, Grath-Chong ME, Chan CT. Quotidian nocturnal hemodialysis improves cytokine profile and enhances erythropoietin responsiveness. ASAIO J 2005; 51: 236-241, doi: 10.1097/ 01.MAT.0000160578.43422.60.

21. Suri R, Depner TA, Blake PG, Heidenheim AP, Lindsay RM. Adequacy of quotidian hemodialysis. Am J Kidney Dis 2003; 42: 42-48, doi: 10.1016/S0272-6386(03)00537-7.

22. Heidenheim AP, Muirhead N, Moist L, Lindsay RM. Patient quality of life on quotidian hemodialysis. Am J Kidney Dis 2003; 42: 36-41, doi: 10.1016/S0272-6386(03)00536-5.

23. Vos PF, Zilch O, Jennekens-Schinkel A, Salden M, Nuyen $\mathrm{J}$, Kooistra MM, et al. Effect of short daily home haemodialysis on quality of life, cognitive functioning and the electroencephalogram. Nephrol Dial Transplant 2006; 21: 2529-2535, doi: 10.1093/ndt/gfl256.

24. Hall YN, Larive B, Painter P, Kaysen GA, Lindsay RM, Nissenson AR, et al. Effects of six versus three times per week hemodialysis on physical performance, health, and functioning: Frequent Hemodialysis Network (FHN) randomized trials. Clin J Am Soc Nephrol 2012; 7: 782-794, doi: 10.2215/CJN.10601011.

25. Finkelstein FO, Schiller B, Daoui R, Gehr TW, Kraus MA, Lea J, et al. At-home short daily hemodialysis improves the long-term health-related quality of life. Kidney Int 2012; 82: 561-569, doi: 10.1038/ki.2012.168.

26. KDOQI Clinical Practice Guideline and Clinical Practice Recommendations for anemia in chronic kidney disease: 2007 update of hemoglobin target. Am J Kidney Dis 2007; 50: 471-530, doi: 10.1053/j.ajkd.2007.06.008.

27. Benz RL, Pressman MR, Hovick ET, Peterson DD. A preliminary study of the effects of correction of anemia with recombinant human erythropoietin therapy on sleep, sleep disorders, and daytime sleepiness in hemodialysis patients (The SLEEPO study). Am J Kidney Dis 1999; 34: 10891095, doi: 10.1016/S0272-6386(99)70015-6. 